\title{
Vaccinating against Helicobacter pylori infection
}

\author{
Steven J. Czinn and Thomas Blanchard
}

\begin{abstract}
Helicobacter pylori infection of the gastric mucosa remains a cause of significant morbidity and mortality almost 30 years after its discovery. H. pylori infection can lead to several gastric maladies, including gastric cancer, and although antimicrobial therapies for the infection exist, the cost of treatment for gastric cancer and the prognosis of individuals who present with this disease make vaccine development a cost effective alternative to bacterial eradication. Experimental mucosal and systemic $H$. pylori vaccines in mice significantly reduce bacterial load and sometimes provide sterilizing immunity. Clinical trials of oral vaccines consisting of $H$. pylori proteins with bacterial exotoxin adjuvants or live attenuated bacterial vectors expressing $H$. pylori proteins induce adaptive immune mechanisms but fail to consistently reduce bacterial load. Clinical trials and murine studies demonstrate that where $H$. pylori is killed, either spontaneously or following vaccination, the host demonstrated cellular immunity. Improved efficacy of vaccines may be achieved in new trials of vaccine formulations that include multiple antigens and use methods to optimize cellular immunity. Unfortunately, the industrial sponsors that served as the primary engine for much of the previous animal and human research have withdrawn their support. A renewed or expanded commitment from the biotechnology or pharmaceutical industry that could exploit recent advances in our understanding of the host immune response to $H$. pylori is necessary for the advancement of an $H$. pylori vaccine.
\end{abstract}

Czinn, S. J. \& Blanchard, T. Nat. Rev. Gastroenterol. Hepatol. 8, 133-140 (2011); published online 8 February 2011; doi:10.1038/nrgastro.2011.1

\section{Introduction}

Helicobacter pylori is a Gram-negative, microaerophilic bacterium that infects the stomach of more than $50 \%$ of the global population and represents a major cause of gastroduodenal pathologies, such as gastritis, gastric and duodenal ulcers, and gastric cancer. ${ }^{1-5}$ Studies have also linked $H$. pylori infection to diverse extragastric diseases of the head and neck, cardiovascular, hepatobiliary, dermatological, immunological, pulmonary and hematological systems. ${ }^{6}$ Although some of these associations remain controversial or are complicated by confounding factors, others have been well demonstrated. The prevalence of $H$. pylori infection ranges from $10-60 \%$ in Western countries and can approach $100 \%$ in developing countries. ${ }^{7}$ Socioeconomic factors have a significant influence on infection rates. ${ }^{7.8} \mathrm{~A}$ US survey performed in 2005 and based on data collected between 1988 and 1991 demonstrated an overall prevalence of $H$. pylori infection of $32.7 \%$ in the general population. ${ }^{8}$ However, this value rose to $48 \%$ for individuals living below the poverty line, and to above $50 \%$ for individuals living in overcrowded homes or those without an education. ${ }^{8}$ The vast majority of $H$. pylori infections are acquired during childhood and the most frequent route of infection is oral-to-oral transmission. ${ }^{7}$ Unless successfully eradicated either by antimicrobial treatment or via host inflammatory and immune responses, most infections persist for life. While

Competing interests

The authors declare no competing interests. the majority of patients remain asymptomatic, $10-20 \%$ are at risk of developing peptic ulcer disease, $1 \%$ will develop gastric adenocarcinoma, and $<1 \%$ will develop mucosa-associated lymphoid tissue lymphoma (MALT). ${ }^{9}$ On the basis of these data, $H$. pylori is classified as a definite class I carcinogen by the WHO. ${ }^{5}$

Antimicrobial therapies for $H$. pylori infection have been very successful, achieving eradication rates of $\geq 90 \%$. ${ }^{2}$ Much of the world, however, would benefit greatly from targeted or widespread vaccination strategies against this infection. Effective treatment of an $H$. pylori infection requires multidrug regimens, typically several antibiotics used in combination with a PPI. ${ }^{2}$ Treatment must be taken several times a day for at least 7 days. Patient compliance with such complex regimens can, therefore, be difficult to maintain. Additionally, antibiotic resistance to frequently used antibiotics, such as clarithromycin and metronidazole, has become an important problem in some countries. ${ }^{10}$ Furthermore, the successful eradication of $H$. pylori infection through antimicrobial therapy does not provide continued protection against the infection. In some countries the rate of reinfection in patients who have achieved initial successful eradication is as high as $15-30 \%$ per year. ${ }^{10-12}$

Perhaps most importantly, most individuals with H. pylori infection who develop gastric cancer typically remain asymptomatic until the cancer becomes advanced, leading to poor prognosis. Proposals to simply administer antimicrobial therapy to individuals
Department of

Pediatrics, Division of Gastroenterology, University of Maryland School of Medicine, 655 W. Baltimore Street, Bressler Research Building, 13-043, Baltimore, MD 21201, USA (S. J. Czinn, T. Blanchard),

Correspondence to:

T. Blanchard

tblanchard@ peds.umaryland.edu 


\section{Key points}

- Many vaccine formulations and strategies have been tested against Helicobacter pylori in mice resulting in significant reductions in bacterial load

- Clinical trials of several different experimental mucosal vaccine formulations have been shown to induce some immune responsiveness, but most fail to induce protective immunity against $\mathrm{H}$. pylori

- Some studies in which bacterial load is reduced following vaccination with candidate vaccines against $H$. pylori, or even sham vaccines, indicate that the host has the potential to eradicate $H$. pylori

- The natural host response to $H$. pylori infection includes the production of regulatory T cells that may limit the host's ability to eradicate $H$. pylori

- New vaccine strategies designed to bypass or override the host immunoregulatory response may provide the best opportunity to develop an efficacious vaccine against $H$. pylori

- Increased participation by the biotechnology or pharmaceutical industries would greatly improve the rate and likelihood of successful $H$. pylori vaccine development the study of candidate vaccine formulations. A survey of studies of candidate vaccines reveals that it is possible to induce some level of immunity against $H$. felis or $H$. pylori infection by use of any one of a number of various vaccination strategies. ${ }^{18}$ Significant reductions in bacterial load, if not sterilizing immunity, can be achieved in vaccinated mice following challenge of their immune system with $H$. pylori or $H$. felis organisms. ${ }^{18}$

When the results of these rodent studies are combined, several important summations can be made that may be applicable to humans. First, immunity can be induced through the use of any one or a combination of a number of different $H$. pylori antigens, including those that are not normally immunodominant during infection. In addition to inactivated whole cell preparations or whole cell lysate, $H$. pylori proteins as diverse as the urease enzyme, cytotoxin-associated gene $\mathrm{A}$ product (CagA), vacuolating toxin A (VacA), catalase, heat shock protein, and others have all been shown to confer immunity against gastric $H$. pylori infection. ${ }^{18}$ Second, vaccination of mice against $H$. pylori is shown to be equally effective when administered therapeutically as prophylactically. ${ }^{19-21}$ Third, immune protection can be induced by many different routes of mucosal vaccination including orogastric, intranasal, and rectal. ${ }^{22-28}$ Of note, vaccination is as efficacious when delivered by systemic routes, such as subcutaneous or intraperitoneal injection, as when delivered topically to mucosal tissue..$^{29-31}$ Although it is surprising that systemic vaccination can induce protection against a strict mucosal pathogen, this finding may prove important given the limitations of mucosal vaccination, which are explored later in this article. Finally, studies in mice have demonstrated that immunity to $H$. pylori infection relies more on developing a strong T-helper-cell $\left(\mathrm{T}_{\mathrm{H}}\right)$-dependent, cellular inflammatory response $\mathrm{e}^{32-36}$ than on a humoral immune response. ${ }^{36,37}$ This finding negates the necessity of limiting candidate vaccines to specific proteins on the surface of the bacteria.

The limitations of murine studies should, however, be kept in mind when applying their findings to potential clinical applications. Although sterilizing immunity against $H$. pylori has been reported on occasion, most studies of murine models have measured immune protection as a significant reduction in bacterial load. Vaccination of humans, therefore, attempts to achieve a result that has been elusive in animal models. Furthermore, many of the problems associated with mucosal vaccination are easily overcome in mice and are likely to be much more problematic in humans. For example, oral vaccination is notoriously weak or nonimmunogenic and requires a mucosal adjuvant or delivery vehicle to circumvent this problem. Mucosal adjuvants are additives that increase the immunogenicity of a candidate vaccine when administered to mucosal surfaces by either orogastric, rectal, or intranasal routes. Few mucosal adjuvants have been identified and although bacterial exotoxins such as cholera toxin (CT) or the closely related Escherichia coli heat labile toxin (LT) can be administered in mice with little untoward side effects, 
humans have proven to be much more sensitive to these proteins, even at similar doses, and adverse events are regularly reported.

\section{Vaccination in large animal models}

The murine model of $H$. pylori infection continues to be important for exploring the use of novel vaccine formulations. Larger animal models, however, have also been employed and these models, although generating some positive results, may be more predictive of the difficulties to be overcome in inducing protective immunity against H. pylori in humans. For example, in one study, the therapeutic vaccination of mice against $H$. pylori resulted in $70 \%$ of mice remaining free of infection 3 months after vaccination. ${ }^{24}$ By contrast, a similar strategy in ferrets infected with the closely related, indigenous Helicobacter mustelae achieved protective immunity in $30 \%$ of the animals. ${ }^{38}$ These data, despite further demonstrating the ability to positively influence the immune response to Helicobacter organisms, suggest that the success achieved in mice might not easily transfer to larger organisms. This study may also indicate that eradicating gastric Helicobacter infections may be more difficult for indigenous infections such as $H$. mustelae in ferrets or $H$. pylori in humans. Indeed, several early studies performed in primate monkey models have demonstrated mixed results that are disappointing when compared with many mouse model studies.

A study of therapeutic vaccination against endogenous Helicobacter heilmannii in cynomolgus monkeys utilized inactivated $H$. pylori organisms or recombinant $H$. pylori urease protein combined with an LT mucosal adjuvant delivered by various routes. The vaccines were largely ineffective, but when delivered parenterally, or by a combination or oral and parenteral routes, some animals exhibited a reduction in bacterial load. ${ }^{39} \mathrm{~A}$ subsequent study of rhesus macaques assessed the efficacy of prophylactic vaccination against the natural acquisition of $H$. pylori infection. This study used an oral vaccine consisting of recombinant $H$. pylori urease and LT; $69 \%$ of animals given the vaccine became infected with H. pylori compared with $93 \%$ of animals that were given a placebo. ${ }^{40}$ A similar vaccine was given therapeutically to monkeys with $H$. pylori infection but failed to induce immune protection. ${ }^{41}$ Additional studies designed to test different routes of vaccination in rhesus macaques were largely unsuccessful. However, a study of monkeys given an initial mucosal vaccine followed by a parenteral boost did report a 22 -fold reduction in the bacterial load of monkeys given the vaccine compared with those given a sham vaccine. ${ }^{42}$ These studies are consistent with observations made in germ-free piglets in which some reduction in bacterial load was achieved following the administration of either an oral or parenteral $H$. pylori whole cell vaccine. ${ }^{43}$ In retrospect, these studies, combined with the many murine studies demonstrating the difficulty of achieving sterilizing immunity, provided a preview of the great challenges that would confront the development of an efficacious $H$. pylori vaccine for use in humans. Conversely, if success can be achieved in these models, it may prove to be predictive of potential success in humans.

\section{Clinical trials}

Clinical trials with prototype $H$. pylori vaccines began at about the same time as some of the nonhuman primate studies described in this article (Table 1). Not surprisingly, given the nature of most of the animal model studies performed during the 1990s, these clinical trials have predominantly focused on $H$. pylori urease-based vaccines in combination with LT adjuvant delivered orally. The first clinical trial tested the therapeutic efficacy of an $H$. pylori vaccine administered to $H$. pyloripositive individuals. ${ }^{44} 180 \mathrm{mg}, 60 \mathrm{mg}$, or $20 \mathrm{mg}$ doses of $H$. pylori urease were administered in combination with either $5 \mu \mathrm{g}$ or $10 \mu \mathrm{g}$ of LT according to an immunization and booster regime previously shown to be successful in mice. Immunogenicity was determined by measuring the number of urease-specific antibody-producing cells in the blood. Disappointingly, no sterilizing immunity was observed in vaccinated individuals, but a significant reduction in bacterial load was observed in individuals given the $20 \mathrm{mg}$ dose of $H$. pylori urease. Gastric inflammation was unaltered by vaccination. Perhaps more disappointing than the lack of protective immunity was the observation that the LT adjuvant induced diarrhea in over $60 \%$ of individuals after the administration of only one dose. The protocol that employed the $10 \mu \mathrm{g}$ dose of LT was discontinued before completion of the study, but the $5 \mu \mathrm{g}$ dose also induced diarrhea in many individuals.

Establishing a dose of CT or LT at which immunogenicity is maintained in the absence of toxicity has been an elusive goal. Both large and small animals have proven to be more tolerant of these adjuvants when administered in the $5-10 \mu \mathrm{g}$ dose range. ${ }^{18} \mathrm{~A}$ study in humans to assess the effectiveness of smaller doses of LT $(0.1-2.5 \mu \mathrm{g})$ in combination with various doses of $H$. pylori urease demonstrated that $2.5 \mu \mathrm{g}$ of LT was sufficient to achieve immunogenicity, but $50 \%$ of the individuals who received this dose experienced diarrhea ${ }^{45}$ In an effort to ameliorate LT toxicity, another clinical trial tested $H$. pylori urease and LT vaccine combinations delivered rectally as opposed to orally. ${ }^{46}$ In this study, $60 \mathrm{mg}$ of H. pylori urease combined with either $5 \mu \mathrm{g}$ or $25 \mu \mathrm{g}$ of LT was given as an enema to $H$. pylori-negative volunteers three times over the course of 28 days. No adverse events of diarrhea or tenesmus were reported by study participants. An immunologic response to LT was achieved by individuals as evidenced by LT-specific B cells in the blood; however, no immunologic response to $H$. pylori urease was achieved as shown by the lack of urease-specific circulating antibodies or T-cell memory responses.

Current strategies aimed at harnessing the use of LT as a mucosal adjuvant involve the creation of genetically modified derivatives of LT in which amino acid substitutions result in limitation of its enzymatic activity. ${ }^{47,48}$ These novel vaccine adjuvants have been tested in combination with H. pylori CagA, VacA, and urease proteins in murine models of $H$. pylori infection ${ }^{24,47}$ and have also 


\section{REVIEWS}

Table 1 | Clinical trials that have evaluated the immunogenicity and efficacy of Helicobacter pylori vaccines

\begin{tabular}{|c|c|c|c|c|c|c|}
\hline Trial & Type & Antigen & Adjuvant & Carrier & $\begin{array}{l}\text { Vaccination } \\
\text { route }\end{array}$ & Response \\
\hline Michetti et al. ${ }^{44}$ & Therapeutic & Urease & LT & NA & Oral & $\begin{array}{l}\uparrow \text { Anti-H.pylori serum IgA titers } \\
\uparrow \text { Anti-H.pylori PBMC ASCs } \\
\text { Significant reduction in bacterial load }\end{array}$ \\
\hline Banerjee et al. ${ }^{45}$ & NA & Urease & LT & NA & Oral & $\begin{array}{l}\uparrow \text { Anti-H. pylori serum IgA and IgG titers } \\
\uparrow \text { Anti-H. pylori PBMC ASCs in some individuals }\end{array}$ \\
\hline $\begin{array}{l}\text { Sougioultzis } \\
\text { et al. }{ }^{46}\end{array}$ & NA & Urease & LT & NA & Rectal & $\begin{array}{l}\uparrow \text { Anti-H. pylori serum IgA and IgG titers in }<20 \% \text { of individuals } \\
\uparrow H \text {. pylori-specific PBMC IgA ASCs in } 25 \% \text { of individuals }\end{array}$ \\
\hline Kotloff et al. ${ }^{49}$ & Therapeutic & HWC & $\mathrm{LT}_{\mathrm{R} 192 \mathrm{G}}$ & NA & Oral & $\begin{array}{l}\uparrow \text { Anti-H. pylori serum and mucosal IgA and IgG titers in } \\
\text { individuals receiving highest dose of antigen } \\
\uparrow \text { PBM cell proliferative and cytokine response }\end{array}$ \\
\hline DiPetrillo et al. ${ }^{52}$ & NA & Urease & NA & $\begin{array}{l}\text { Salmonella } \\
\text { enterica serovar } \\
\text { Typhi Ty1033 }\end{array}$ & Oral & No H. pylori-specific immune response detected \\
\hline $\begin{array}{l}\text { Malfertheiner } \\
\text { et al. }\end{array}$ & NA & $\begin{array}{l}\text { VacA, } \\
\text { CagA, } \\
\text { NAP }\end{array}$ & $\begin{array}{l}\text { Aluminum } \\
\text { hydroxide }\end{array}$ & NA & Intramuscular & $\begin{array}{l}\uparrow \text { Serum lgG to all three proteins in } 86 \% \text { of individuals } \\
\uparrow \text { PBMC cell cytokine response to CagA and VacA proteins } \\
\text { Strong humoral and cellular memory response upon repeated } \\
\text { vaccination after } 18 \text { months }\end{array}$ \\
\hline
\end{tabular}

Abbreviations: ASC, antivbody-secreting cell; CagA, cytotoxin-associated gene A product; HWC, Helicobacter pylori whole cell; IgA, immunoglobulin A; IgG, immunoglobulin G; LT, Escherichia coli heat labile toxin; NA, not applicable/available?; NAP, neutrophil-activating protein; PBM, peripheral blood mononuclear cells; VacA, vacuolating toxin A.

been evaluated in limited clinical studies that have used inactivated whole cell $H$. pylori preparations as antigens. ${ }^{49}$ A genetically modified formulation of LT in combination with a whole cell $H$. pylori preparation might be expected to be more immunogenic than other vaccine candidates since its multivalency, complexity, and particulate nature should lead to a strong immune response. ${ }^{50}$ In a clinical study, $H$. pylori-infected or uninfected individuals received an oral vaccine of formalin-inactivated $H$. pylori organisms plus $25 \mu \mathrm{g} \mathrm{LT}_{\mathrm{R} 192 \mathrm{G}}$, a mutant form of LT with an amino acid substitution in the trypsin cleavage site that results in reduced toxicity. ${ }^{49} \mathrm{H}$. pylori-specific salivary and fecal antibody responses were increased in individuals already infected with $H$. pylori and who received the highest dose of vaccine $\left(2.5 \times 10^{10}\right.$ whole cells $)$. Peripheral blood lymphocyte memory immune responses were only observed in uninfected individuals. These data support the notion that a nontoxic derivative of LT can induce immunogenicity against $H$. pylori.

This vaccine was also tested for its efficacy as a therapeutic agent. $H$. pylori-positive individuals were given whole cell $H$. pylori preparations or placebos in combination with $\mathrm{LT}_{\mathrm{R} 192 \mathrm{G}}$. The noninvasive ${ }^{13} \mathrm{C}$ urea breath test was used to detect colonization. None of the individuals in this study were shown to have eradicated $H$. pylori when evaluated up to 7.5 months after vaccination. It is impossible to determine if the bacterial load in these individuals was reduced or not as colony counts were not determined and the ${ }^{13} \mathrm{C}$ urea breath test cannot provide a quantitative evaluation. The mutant LT did, however, induce diarrhea in $18 \%$ of vaccinated individuals. The observed toxicity may be ameliorated with reduced doses of the mutant LT. These studies demonstrate a need for continued development of nontoxic mucosal adjuvants. In 2010, a novel double mutant of LT was evaluated as part of a vaccine against $H$. pylori in mice and was shown to be as immunogenic and efficacious as the single mutant $\mathrm{LT}_{\mathrm{R} 192 \mathrm{G}}{ }^{48}$

Recombinant bacterial vector vaccines that express H. pylori proteins have also been evaluated for vaccination against $H$. pylori infection in humans. These prototypes have relied on the use of avirulent strains of Salmonella, which is a bacterium that targets the tissue of the small intestine and normally induces host immunity. Studies of this approach have uniformly utilized recombinant expression of the $H$. pylori urease protein and most have focused on immunogenicity in humans. The earliest clinical studies of such vaccines, which used either avirulent Salmonella enterica serovar Typhi or Salmonella enterica serovar Typhimurium expressing H. pylori urease, were disappointing. ${ }^{51,52}$ Only one of these formulations was found to induce any ureasespecific antibody response and even this response varied between individuals.

A subsequent study of an oral vaccine using Salmonella strain Ty21a expressing both the A and B subunits of $H$. pylori urease demonstrated only weak immune responses in $H$. pylori-negative individuals. None of the individuals who received the oral vaccine responded with a urease-specific serum antibody response, although several patients did develop a T-cell memory response. ${ }^{53}$ The group who performed this research carried out a 
subsequent study to determine if previous exposure to Salmonella Ty21a might influence the host response to a recombinant Salmonella Ty21a strain expressing A and B subunits of $H$. pylori urease; however, vaccination with the recombinant Salmonella Ty21a strain following initial exposure to Salmonella Ty21a had no influence on host response..$^{54}$ Individuals failed to develop a humoral immune response to $H$. pylori urease although, similar to the previous study, many individuals developed a urease-specific T-cell memory response.

The recombinant Salmonella Ty21a vaccine was evaluated in a clinical challenge study that examined $H$. pylori-negative individuals. ${ }^{55}$ Participants received multiple doses of either the $H$. pylori urease-expressing Salmonella strain or another recombinant strain of this bacterium expressing the HP0231 H. pylori antigen. Individuals were challenged with a human strain of $H$. pylori that had been previously characterized for use in these types of studies. ${ }^{56}$ Individuals developed immune responses typically associated with $H$. pylori infection, such as increased serum cytokine levels and histologic gastritis. ${ }^{55}$ The challenge also resulted in an increase in urease-specific, peripheral blood, antibody-secreting cell numbers that had not been observed before the challenge. Although bacterial load decreased in 8 of 33 vaccinated individuals, this finding also occurred in 5 of 25 control individuals who were administered a Salmonella Ty21a control vector. The recombinant attenuated vaccines were well tolerated by individuals in these studies. It is possible that improved success might be achieved through the use of additional $H$. pylori protein antigens to create multivalent recombinant vaccine strains.

\section{Positive implications of research}

It is difficult to describe the vaccine trials discussed in this article as anything other than disappointing. In fact, there is no indication that any of these experimental vaccines are in continued development for additional human studies. However, it would be a mistake to dismiss the value of these studies, or to admit the futility of developing an efficacious $H$. pylori vaccine. Certain aspects of these studies, combined with immunologic studies in mice and humans, indicate why vaccine development has been so difficult in this area and may provide clues as to how vaccination against $H$. pylori could be improved. First, any study in which $H$. pylori-specific humoral or cellular immune responses are induced is evidence that host immunity can be positively influenced through these vaccine technologies. ${ }^{44-46,49,55}$ Second, it is worth noting that Michetti et al. recorded a significant reduction in bacterial load in a group of patients who had received vaccination with $H$. pylori urease plus LT. ${ }^{44}$ Aebischer et al. also noted decreased bacterial loads after vaccinating individuals with a recombinant Salmonella Ty21a H. pylori vaccine..$^{55}$ While this reduction in bacterial load occurred in both vaccinated and placebo groups, the reduction in bacterial load was associated with a strong cell-mediated immune response and the underlying observation remains the same: the host is capable under certain circumstances, either through vaccine-induced or through natural host response pathways, of eliminating $H$. pylori from the gastric mucosa, even if sterilizing immunity cannot be achieved. Improved understanding of why the host normally fails to eradicate $H$. pylori during natural infection may help design improved experimental vaccines.

Investigators have long sought to understand why the host fails to protect itself from chronic $H$. pylori infection. Our current understanding of immunoregulation at mucosal tissues suggests that the host may actively suppress inflammatory and immune responses to $H$. pylori. Mucosal tissues, such as the colon and respiratory tract, are tightly regulated to control the host response to environmental antigens and microbes. This regulation is accomplished by, among other mechanisms, regulatory $\mathrm{T}\left(\mathrm{T}_{\mathrm{REG}}\right)$ cells. ${ }^{57}$ Although the plasticity and functions of $\mathrm{T}_{\mathrm{REG}}$ cells are still actively being discerned, $\mathrm{T}_{\mathrm{REG}}$ cells, identified by increased levels of CD25 expression and the FoxP3 transcription factor, have an active role in suppressing autoimmunity and certain immune responses at the mucosa. $\mathrm{T}_{\mathrm{REG}}$-mediated control at environmental interfaces seems to be meditated by the production of interleukin 10 (IL-10). ${ }^{58}$ Mouse models of $H$. pylori infection have demonstrated that in the absence of IL-10, H. pylori-associated inflammation increases significantly and the bacteria are eradicated. ${ }^{34,59}$ Studies in which immunodeficient mice are reconstituted with T cells lacking CD25 and then challenged with $H$. pylori organisms show that the mice respond to infection with increased gastritis and reduced bacterial load. ${ }^{60}$ Antibody-mediated depletion of CD $25^{+} \mathrm{T}$ cells in such mice similarly results in increased inflammation and reduced bacterial numbers. ${ }^{61}$ Our own murine studies in which $\mathrm{T}_{\mathrm{REG}}$ cell engagement is blocked in vivo through CTLA-4 specific antibodies, or phagocyteoxidase-deficient mice are used that are known to have limited numbers of $\mathrm{T}_{\mathrm{REG}}$ cells, show that $H$. pylori infection involves a robust local inflammatory response and a corresponding reduction in $H$. pylori numbers. ${ }^{62,63}$

Evidence also exists for the involvement of $\mathrm{T}_{\mathrm{REG}}$ cells in the response of humans to $H$. pylori. The in vitro $H$. pylori-specific memory response of blood or gastric T cells to $H$. pylori infection in $H$. pylori-positive individuals is not significantly greater than that of lymphocytes in H. pylori-negative individuals. ${ }^{64-68}$ Furthermore, in humans, the depletion of $\mathrm{CD} 25^{+} \mathrm{T}$ cells before in vitro stimulation with $H$. pylori antigens results in a significantly stronger interferon (IFN) $\gamma$ response. ${ }^{69}$ In addition, biopsy samples from the gastric mucosa of patients with $H$. pylori infection have revealed the presence of $\mathrm{T}_{\mathrm{REG}}$ cells. Harris et al. reported that children infected with $H$. pylori possess greater numbers of $\mathrm{T}_{\mathrm{REG}}$ cells in the gastric mucosa and display significantly less gastritis than infected adults. ${ }^{70}$ The host, therefore, may recognize $H$. pylori as nondangerous bacteria and engage the same types of immunoregulation that occur in the intestinal tract in response to commensal bacteria. Although histologic gastritis is always present in individuals with H. pylori infection, ${ }^{71}$ it is important to remember that over $80 \%$ of patients remain asymptomatic and otherwise 


\section{REVIEWS}

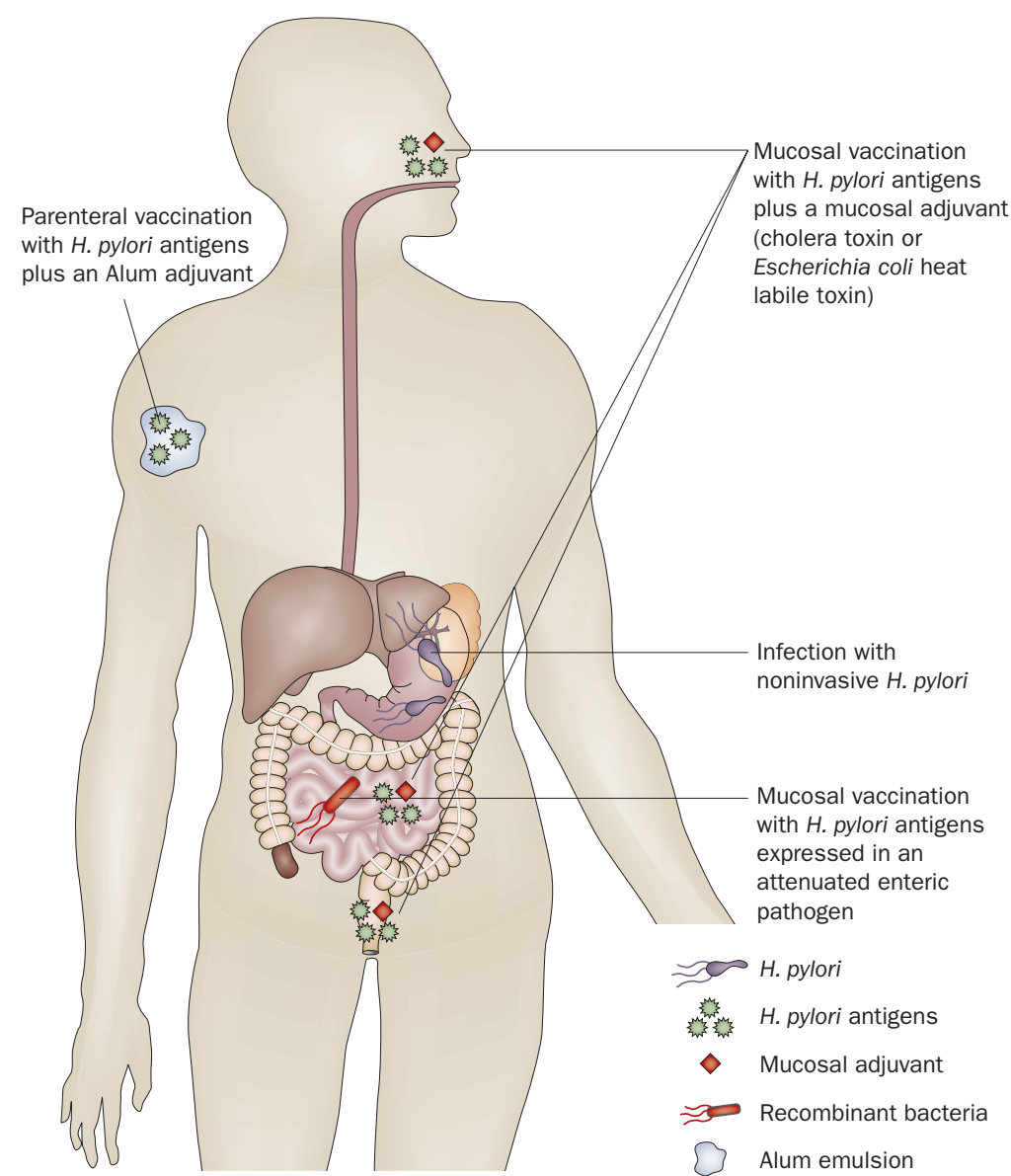

Figure 1 | Illustration of the most commonly tested Helicobacter pylori vaccination strategies and routes in human and animal models. Most vaccine strategies induce T-helper $\left(T_{H}\right)$ cell responses characterized by production of interferon $\gamma$ and/or interleukin 17. Infection with $\mathrm{H}$. pylori may also induce this type of response but also results in a strong regulatory $\mathrm{T}$-cell response that suppresses the $\mathrm{T}_{H}$ cell activity.

healthy. This predisposition of the host to downregulate immune responses at mucosal surfaces must be overcome in order to improve the efficacy of an $H$. pylori vaccine (Figure 1).

\section{Challenges}

The continued development of a vaccine for H. pylori will most likely progress very slowly. At least three challenges must be overcome in order to make meaningful progress. First, the selection of vaccine antigens should be addressed in order to achieve an immunologically complex vaccine that elicits strong antibody responses and, more importantly, a strong T-cell memory response. Evidence from mouse models shows that although antibodies may have a role in vaccine-mediated immunity, they are not required to elicit immune protection. ${ }^{21,36,37}$ Conversely, in most murine studies of $H$. pylori vaccines in which $H$. pylori numbers are reduced either spontaneously or through vaccination, local inflammation or cellular immune responses are significantly increased. In mouse models, an inverse correlation between degree of inflammation and level of bacterial colonization in the context of both vaccination-based inflammation and immune dysregulation has been made by multiple laboratories. ${ }^{27,59-63,72}$ Such a correlation was also shown in the study of the Salmonella Ty21a-based recombinant H. pylori vaccine. ${ }^{55}$ While some researchers believe this increase in inflammatory activity is detrimental to the host, it is more likely that it is essential to the protection of the host as the stomach normally lacks a resident white blood cell pool and these cells are necessary for immune activity. Evidence suggests that when bacteria are completely eradicated gastritis eventually dissipates. ${ }^{34,72}$ Increased efficacy of $H$. pylori vaccination has been associated with the use of multiple antigens in mice. ${ }^{50} \mathrm{It}$ is noteworthy that studies in humans to test the immunogenicity and safety of a systemic vaccine against $H$. pylori have reported strong memory immune responses with the use of a trivalent vaccine consisting of CagA, VacA, and neutrophil-activating protein (NAP) (Table 1). ${ }^{73}$

Second, as mentioned earlier, it will be necessary to design vaccines or vaccine delivery systems that effectively override or bypass the downregulatory mechanisms of the gastrointestinal tract. This may be accomplished through continued development of CT or LT genetic mutants or through continued advances in attenuated live bacterial vaccine delivery vehicles. However, the use of systemic vaccination may provide the most straight forward means of inducing potent, proinflammatory $\mathrm{T}$-cell responses in the absence of a dominant $\mathrm{T}_{\mathrm{REG}}$ cell response that can then be recruited to the gastric mucosa upon $H$. pylori infection. We and others have demonstrated that systemic vaccination with aluminum-based adjuvants are at least as efficacious as experimental mucosal vaccines in mice. ${ }^{29-31}$ The aforementioned trivalent vaccine that uses CagA, VacA, and NAP in combination with an FDA-approved aluminum hydroxide adjuvant has been delivered by intramuscular injection to $H$. pylori-infected Beagle dogs and was shown to reduce bacterial colonization and gastritis. ${ }^{74}$ Animal models indicate that protective immunity against H. pylori involves a strong $\mathrm{T}_{\mathrm{H}} 1$ and/or $\mathrm{T}_{\mathrm{H}} 17$ cell response that promotes a mixed lymphocyte and granulocyte response in the gastric mucosa. ${ }^{32-34,75}$ Inducing these types of $H$. pylori-specific T cells is the crucial step in generating immunity to this infection and it may not matter whether this is accomplished by mucosal or systemic vaccination.

Finally, success in this arena cannot be accomplished without greater involvement from the biotechnology and pharmaceutical industries. Small laboratory groups in universities and medical institutes have been instrumental in performing much of the pioneering work in animal models on the selection of antigens, routes of vaccination, the nature of the protective immune response, and the screening of many different adjuvants and delivery systems of vaccination. However, the generation of safe and well-characterized vaccines in the scale necessary to perform large studies, and the capital investment required to carry out meaningful clinical trials can only be accomplished by, or in cooperation with, industry. Past studies of $H$. pylori vaccines have included support from companies such as Antex Biologics (Gaithersburg, MD), 
AstraZeneca (Boston, MA), Avant Immunotherapeutics (Needham, MA), OraVax, now Acambis (Cambridge, MA), Chiron Corporation, now Novartis International AG (Basel, Switzerland), CSL (Melbourne, Australia), and Pasteur Merieux Connaught (Marcy l'Etoile, France) among others. Most of these companies no longer have $H$. pylori vaccine initiatives. The lack of support has resulted in relatively few clinical studies being performed in the past few years. Indeed, the only ongoing clinical study that we have knowledge of is the trivalent, systemic vaccine, which has now been tested for safety and immunogenicity through research funded by Novartis. ${ }^{73}$ Given the complex nature of $H$. pylori infection and the many challenges that confront vaccine development, progress will require an increased commitment from private industry. The need for such commitment will only increase if the current vaccine being investigated by Novartis lacks acceptable efficacy.

\section{Conclusions}

The flurry of research into $H$. pylori vaccine development that occurred in animal models throughout the 1990s led to the performance of several clinical trials by the end of that decade and throughout the 2000s. H. pylori vaccine strategies have predominantly included oral delivery of either whole cell or subunit vaccines in combination with cholera toxin or LT derivatives, oral delivery of attenuated, recombinant bacteria expressing $H$. pylori antigens, and more recently intramuscular delivery of an $H$. pylori subunit vaccine with an aluminum hydroxide adjuvant. Although some immune responsiveness has been demonstrated by these vaccines, no study has reported protective immunity. Recent studies in animal models suggest that the host immune response to $H$. pylori contains a significant $\mathrm{T}_{\mathrm{REG}}$ cell component that may limit immune and inflammatory mechanisms at the gastric mucosa. Vaccination strategies that successfully overcome this regulation and induce strong, proinflammatory $\mathrm{T}_{\mathrm{H}}$ cell responses may provide the best strategy for achieving acceptable levels of immune protection against $H$. pylori. The lack of participation or support from industrial partners in the past few years has resulted in a paucity of vaccine trials. Increased support from biotechnology companies and the pharmaceutical industry would provide a much needed boost for new vaccine initiatives and enable clinical trials to be conducted that take advantage of new knowledge gained in recent years about host mechanisms that are involved in bacterial clearance.

Review criteria
The PubMed database was searched using various
combinations of the terms "Helicobacter pylori",
"Helicobacter felis", "vaccine" (and derivative words),
"immunize" (and derivative words), "adjuvant", "mucosal”,
"mouse", "murine", "animal", "nonhuman primate",
"clinical trial", and "safety" to identify articles published
between 1983 and 2010. Full-length articles published in
English were selected. For murine and clinical vaccine
studies, the reference lists of recent reviews and research
reports were used to identify further articles. The
publication histories of well known experts in the fields of
$H$. pylori immunology and regulatory T cells were searched
to identify additional relevant articles.

1. Eurogast Study Group. An international association between Helicobacter pylori infection and gastric cancer. Lancet 341, 1359-1362 (1993).

2. NIH Consensus Conference. Helicobacter pylori in peptic ulcer disease. J. Am. Med. Assn. 272, 65-69 (1994).

3. Parsonnet, J. et al. Helicobcter pylori infection and the risk of gastric carcinoma. N. Engl. J. Med. 325, 1127-1131 (1991).

4. Warren, J. R. \& Marshall, B. J. Unidentified curved bacilli on gastric epithelium in active chronic gastritis. Lancet 1, 1273-1275 (1983).

5. WHO. in IARC monographs on the carcogenic risks to humans. 61, 177-241 (IARC, Lyon, 1994).

6. Franceschi, F. \& Gasbarrini, A. Helicobacter pylori and extragastric diseases. Best Pract. Res. Clin. Gastroenterol. 21, 325-334 (2007).

7. Graham, D. Y. et al. Epidemiology of Helicobacter pylori in an asymptomatic population in the United States. Effect of age, race, and socioeconomic status. Gastroenterology 100 , 1495-1501 (1991).

8. Kruszon-Moran, D. \& McQuillan, G. M. Seroprevalence of six infectious diseases among adults in the United States by race/ ethnicity: data from the third national health and nutrition examination survey. Adv. Data 352, 1-9 (2005).

9. Luther, J. \& Kao, J. Y. Considering global vaccination against Helicobacter pylori. South. Med. J. 103, 185-186 (2010).
10. Frenck, R. W. Jr \& Clemens, J. Helicobacter pylori in the developing world. Microbes Infect. 8, 705-713 (2003).

11. Parsonnet, J. What is the Helicobacter pylori global reinfection rate? Can. J. Gastroenterol. 17 (Suppl. B), 46-48 (2003).

12. Gisbert, J. The recurrence of Helicobacter pylori infection: incidence and variables influencing it. A critical review. Am. J. Gastroenterol. 100, 2083-2099 (2005).

13. Rupnow, M. F., Shachter, R. D., Owens, D. K. \& Parsonnet, J. Quantifying the population impact of a prophylactic Helicobacter pylori vaccine. Vaccine 20, 879-885 (2001).

14. Svennerholm, A. M. \& Lundgren, A. Progress in vaccine development against Helicobacter pylori. FEMS Immunol. Med. Microbiol. 50, 146-156 (2007).

15. Marshall, B. J. \& Warren, J. R. Unidentified curved bacilli in the stomach of patients with gastritis and peptic ulceration. Lancet 1, 1311-1315 (1984).

16. Lee, A., Fox, J. G., Otto, G. \& Murphy, J. A small animal model of human Helicobacter pylori active chronic gastritis. Gastroenterology 99 , 1315-1323 (1990).

17. Lee, A. et al. A standardised mouse model of Helicobacter pylori infection. Introducing the Sydney strain. Gastroenterology 112, 1386-1397 (1997).

18. Blanchard, T. G. \& Nedrud, J. G. in Helicobacter pylori in the $21^{\text {st }}$ century. $1^{\text {st }}$ edn Ch. 9 (eds Sutton, P. \& Mitchell, H.) 167-190 (CABI, Wallingford, 2010).
19. Corthesy-Theulaz, I. et al. Oral immunization with Helicobacter pylori urease B subunit as a treatment against Helicobacter infection in mice. Gastroenterology 109, 115-121 (1995).

20. Doidge, C. et al. Therapeutic immunisation against Helicobacter infection. Lancet 343, 914-915 (1994).

21. Sutton, P. et al. Therapeutic immunization against Helicobacter pylori infection in the absence of antibodies. Immunol. Cell. Biol. 78 28-30 (2000).

22. Chen, M. et al. Immunisation against gastric infection with Helicobacter species: first step in the prophylaxis of gastric cancer? Zentralbl. Bakteriol. 280, 155-165 (1993).

23. Czinn, S. J., Cai, A. \& Nedrud, J. G. Protection of germ-free mice from infection by Helicobacter felis after active oral or passive IgA immunization. Vaccine 11, 637-642 (1993).

24. Ghiara, P et al. Therapeutic intragastric vaccination against Helicobacter pylori in mice eradicates an otherwise chronic infection and confers protection against reinfection. Infect. Immun. 65, 4996-5002 (1997).

25. Lee, C. K. et al. Oral Immunization with recombinant Helicobacter pylori urease induces secretory IgA antibodies and protects mice from challenge with Helicobacter felis. J. Infect. Dis. 172, 161-172 (1995).

26. Marchetti, M. et al. Development of a mouse model of Helicobacter pylori infection that mimics human disease. Science 267 , 1655-1658 (1995). 
27. Michetti, P. et al. Immunization of BALB/c mice against Helicobacter felis infection with Helicobacter pylori urease. Gastroenterology 107, 1002-1011 (1994).

28. Weltzin, R. et al. Novel intranasal immunization techniques for antibody induction and protection of mice against gastric Helicobacter felis infection. Vaccine 15, 370-376 (1997).

29. Eisenberg, J. C. et al. Protective efficacy of antiHelicobacter pylori immunity following systemic immunization of neonatal mice. Infect. Immun. 71, 1820-1827 (2003).

30. Gottwein, J. M. et al. Protective anti-Helicobacter immunity is induced with aluminum hydroxide or complete Freund's adjuvant by systemic immunization. J. Infect. Dis. 184, 308-314 (2001).

31. Guy, B. et al. Systemic immunization with urease protects mice against Helicobacter pylor infection. Vaccine 16, 850-856 (1998).

32. Delyria, E. S., Redline, R. W. \& Blanchard, T. G. Vaccination of mice against $H$. pylori induces a strong Th-17 response and immunity that is neutrophil dependent. Gastroenterology 136, 247-256 (2008).

33. Velin, D. et al. Interleukin-17 is a critical mediator of vaccine-induced reduction of Helicobacter infection in the mouse model. Gastroenterology 136, 2237-2246 (2009).

34. Eaton, K. A., Mefford, M. \& Thevenot, T. The role of $T$ cell subsets and cytokines in the pathogenesis of Helicobacter pylori gastritis in mice. J. Immunol. 166, 7456-7461 (2001).

35. Velin, D., Bachmann, D., Bouzourene, H. \& Michetti, P. Mast cells are critical mediators of vaccine-induced Helicobacter clearance in the mouse model. Gastroenterology 129, 142-155 (2005).

36. Ermak, T. H. et al. Immunization of mice with urease vaccine affords protection against Helicobacter pylori infection in the absence of antibodies and is mediated by MHC class IIrestricted responses. J. Exp. Med. 188, 2277-2288 (1998).

37. Blanchard, T. G. et al. Antibody-independent protective mucosal immunity to gastric helicobacter infection in mice. Cell. Immunol. 191, 74-80 (1999).

38. Cuenca, R. et al. Therapeutic immunization against Helicobacter mustelae in naturally infected ferrets. Gastroenterology 110, 1770-1775 (1996).

39. Guy, B. et al. Mucosal, systemic, or combined therapeutic immunizations in cynomolgus monkeys naturally infected with Gastrospirillum hominis-like organisms. Vaccine Research 6 , 141-150 (1997).

40. Dubois, A. et al. Immunization against natural Helicobacter pylori infection in nonhuman primates. Infect. Immun. 66, 4340-4346 (1998).

41. Lee, C. K. et al. Immunization with recombinant Helicobacter pylori urease decreases colonization levels following experimental infection of rhesus monkeys. Vaccine 17 , 1493-1505 (1999).

42. Lee, C. K. et al. Immunization of rhesus monkeys with a mucosal prime, parenteral boost strategy protects against infection with Helicobacter pylori. Vaccine 17, 3072-3082 (1999).

43. Eaton, K. A., Ringler, S. S. \& Krakowka, S. Vaccination of gnotobiotic piglets against Helicobacter pylori. J. Infect. Dis. $\mathbf{1 7 8}$, 1399-1405 (1998).

44. Michetti, P. et al. Oral immunization with urease and Escherichia coli heat-labile enterotoxin is safe and immunogenic in Helicobacter pylori- infected adults. Gastroenterology 116, 804-812 (1999).

45. Banerjee, S. et al. Safety and efficacy of low dose Escherichia coli enterotoxin adjuvant for urease based oral immunisation against Helicobacter pylori in healthy volunteers. Gut 51, 634-640 (2002).

46. Sougioultzis, S. et al. Safety and efficacy of E. coli enterotoxin adjuvant for urease-based rectal immunization against Helicobacter pylori. Vaccine 21, 194-201 (2002).

47. Marchetti, M. et al. Protection against Helicobacter pylori infection in mice by intragastric vaccination with $H$. pylori antigens is achieved using a non-toxic mutant of $E$. coli heatlabile enterotoxin (LT) as adjuvant. Vaccine 16, 33-37 (1998).

48. Summerton, N. A. et al. Toward the development of a stable, freeze-dried formulation of Helicobacter pylori killed whole cell vaccine adjuvanted with a novel mutant of Escherichia coli heat-labile toxin. Vaccine $\mathbf{2 8}, \mathbf{1 4 0 4 - 1 4 1 1}$ (2010).

49. Kotloff, K. L. et al. Safety and immunogenicity of oral inactivated whole-cell Helicobacter pylori vaccine with adjuvant among volunteers with or without subclinical infection. Infect. Immun. 69, 3581-3590 (2001).

50. Ferrero, R. L. et al. The GroES homolog of Helicobacter pylori confers protective immunity against mucosal infection in mice. Proc. Natl Acad. Sci. USA 92, 6499-6503 (1995).

51. Angelakopoulos, H. \& Hohmann, E. L. Pilot study of phoP/phoQ-deleted Salmonella enterica serovar typhimurium expressing Helicobacter pylori urease in adult volunteers. Infect. Immun. 68, 2135-2141 (2000).

52. DiPetrillo, M. D. et al. Safety and immunogenicity of phoP/phoQ-deleted Salmonella typhi expressing Helicobacter pylori urease in adult volunteers. Vaccine 18, 449-459 (1999).

53. Bumann, D. et al. Safety and immunogenicity of live recombinant Salmonella enterica serovar Typhi Ty21a expressing urease $A$ and $B$ from Helicobacter pylori in human volunteers. Vaccine 20, 845-852 (2001).

54. Metzger, W. G. et al. Impact of vector-priming on the immunogenicity of a live recombinant Salmonella enterica serovar typhi Ty21a vaccine expressing urease A and $\mathrm{B}$ from Helicobacter pylori in human volunteers. Vaccine $\mathbf{2 2}$, 2273-2277 (2004).

55. Aebischer, T. et al. Correlation of T cell response and bacterial clearance in human volunteers challenged with Helicobacter pylori revealed by randomised controlled vaccination with Ty21abased Salmonella vaccines. Gut 57, 1065-1072 (2008).

56. Graham, D. Y. et al. Challenge model for Helicobacter pylori infection in human volunteers. Gut 53, 1235-1243 (2004).

57. Sakaguchi, S. et al. Regulatory T cells: how do they suppress immune responses? Int. Immunol. 21, 1105-1111 (2009).

58. Rubtsov, Y. P. et al. Regulatory T cell-derived interleukin-10 limits inflammation at environmental interfaces. Immunity $\mathbf{2 8}$, 546-558 (2008).

59. Matsumoto, Y. et al. Eradication of Helicobacter pylori and resolution of gastritis in the gastric mucosa of IL-10-deficient mice. Helicobacter 10, 407-415 (2005).

60. Raghavan, S. et al. Absence of CD4+CD25+ regulatory $T$ cells is associated with a loss of regulation leading to increased pathology in Helicobacter pylori-infected mice. Clin. Exp. Immunol. 132, 393-400 (2003).
61. Rad, R. et al. CD25+/Foxp3+ T cells regulate gastric inflammation and Helicobacter pylori colonization in vivo. Gastroenterology 131, 525-537 (2006)

62. Anderson, K. M., Czinn, S. J., Redline, R. W. \& Blanchard, T. G. Induction of CTLA-4-mediated anergy contributes to persistent colonization in the murine model of gastric Helicobacter pylori infection. J. Immunol. 176, 5306-5313 (2006).

63. Blanchard, T. G., Yu, F., Hsieh, C. L. \& Redline, R. W. Severe inflammation and reduced bacteria load in murine helicobacter infection caused by lack of phagocyte oxidase activity. J. Infect. Dis. 187, 1609-1615 (2003).

64. Fan, X. J. et al. Gastric T lymphocyte responses to Helicobacter pylori in patients with $\mathrm{H}$. pylori colonisation. Gut 35, 1379-1384 (1994).

65. Karttunen, R. Blood lymphocyte proliferation, cytokine secretion and appearance of T cells with activation surface markers in cultures with Helicobacter pylori. Comparison of the responses of subjects with and without antibodies to H. pylori. Clin. Exp. Immunol. 83, 396-400 (1991)

66. Karttunen, R. Helicobacter pylori induces lymphocyte activation in peripheral blood cultures. Clin. Exp. Immunol. 82, 485-488 (1990).

67. Karttunen, R., Karttunen, T., Ekre, H. P. \& MacDonald, T. T. Interferon gamma and interleukin 4 secreting cells in the gastric antrum in Helicobacter pylori positive and negative gastritis. Gut 36, 341-345 (1995).

68. Sharma, S. A. et al. Humoral and cellular immune recognition of Helicobacter pylori proteins are not concordant. Clin. Exp. Immunol. 97, 126-132 (1994).

69. Raghavan, S., Suri-Payer, E. \& Holmgren, J. Antigen-specific in vitro suppression of murine Helicobacter pylori-reactive immunopathological T cells by CD4CD25 regulatory T cells. Scand. J. Immunol. 60, 82-88 (2004).

70. Harris, P. R. Helicobacter pylori gastritis in children is associated with a regulatory T-cell response. Gastroenterology 134, 491-499 (2008)

71. Dooley, C. P. et al. Prevalence of Helicobacter pylori infection and histologic gastritis in asymptomatic persons. N. Engl. J. Med. 321, 1562-1566 (1989).

72. Garhart, C. A., Redline, R. W., Nedrud, J. G. \& Czinn, S. J. Clearance of Helicobacter pylori infection and resolution of postimmunization gastritis in a kinetic study of prophylactically immunized mice. Infect. Immun. 70, 3529-3538 (2002)

73. Malfertheiner, P. et al. Safety and immunogenicity of an intramuscular Helicobacter pylori vaccine in noninfected volunteers: a phase I study. Gastroenterology 135, 787-795 (2008).

74. Rossi, G. et al. Therapeutic vaccination against Helicobacter pylori in the beagle dog experimental model: safety, immunogenicity, and efficacy. Infect. Immun. 72, 3252-3259 (2004).

75. Sayi, A. et al. The CD4+ T cell-mediated IFNgamma response to Helicobacter infection is essential for clearance and determines gastric cancer risk. J. Immunol. 182, 7085-7101 (2009).

Author contributions

T. G. Blanchard and S. J. Czinn contributed equally to researching data for the article, discussions of content, writing and reviewing/editing of the manuscript before submission. 